\title{
Packaging et chaîne logistique en boucle fermée : contribution au passage d'une économie linéaire à une économie circulaire
}

François Fulconis \& Bernd Philipp

To cite this article: François Fulconis \& Bernd Philipp (2016) Packaging et chaîne logistique en boucle fermée : contribution au passage d'une économie linéaire à une économie circulaire, Logistique \& Management, 24:3-4, 186-198, DOI: 10.1080/12507970.2016.1268936

To link to this article: https://doi.org/10.1080/12507970.2016.1268936

Published online: 09 Jan 2017.

Submit your article to this journal $\sqsubset$

Џ Article views: 76

View Crossmark data $\complement$ 


\title{
Packaging et chaîne logistique en boucle fermée : contribution au passage d'une économie linéaire à une économie circulaire
}

\author{
François Fulconis ${ }^{a, b}$ et Bernd Philipp ${ }^{b, c}$ \\ aUniversité d'Avignon et des Pays de Vaucluse (UAPV), IUT - Département Packaging, Avignon, France ; ${ }^{b}$ CRET-LOG, Aix-Marseille \\ Université (AMU), Aix-en-Provence, France ; cÉcole Supérieure du Commerce Extérieur (ESCE), Paris, France
}

\begin{abstract}
RÉSUMÉ
Depuis le début des années 2000 , la relation forte existant entre le packaging et les autres composants du cycle de vie du produit est reconnue. Parallèlement, dans une perspective de développement durable, un intérêt grandissant est porté à la place qu'occupe le packaging au sein des chaînes logistiques multi-acteurs, et ce, dans des secteurs d'activité très variés. Fort de ce constat, cet article adopte une posture novatrice du packaging : analyser ses fonctions logistiques par rapport à leur capacité à favoriser le passage d'une économie linéaire à une économie circulaire. À partir d'une étude sur les fondements théoriques mobilisables et de la proposition d'un modèle de recherche relatif à un packaging scorecard adapté à une vision circulaire de l'économie, les premiers résultats d'une étude empirique réalisée auprès d'experts de l'industrie du packaging sont présentés.
\end{abstract}

\section{Packaging and closed-loop supply chain: contribution to the transition from a linear economy to a circular economy}

ABSTRACT

The strong relationship between the packaging and the other components of the product life cycle has been fully recognised since the beginning of the millennium. In the same manner, one observes a growing interest, particularly through the lens of sustainable development, in the role that packaging plays within complex supply chains, which is true for many industries. In line with these evolutions, this article adopts a fresh approach of packaging, by analysing its logistics functions vis-à-vis their capacity to promote the transformation of a linear economy into a circular one. The authors (1) carried out a study on the relevant theoretical framework and (2) proposed a research model related to a packaging scorecard that adopts a circular economy perspective, before (3) presenting first results of an empirical study targeting packaging industry experts.

\section{MOTS-CLÉS}

Chaîne logistique en boucle fermé ; développement durable ; économie circulaire ; emballage ; packaging scorecard ; reverse supply chain

\section{KEYWORDS}

Closed-loop supply chain; sustainable development; circular economy; packaging; packaging scorecard; reverse supply chain

\section{Introduction ${ }^{1}$}

Selon l'Ellen MacArthur Foundation (2013), un consommateur des pays de l'OCDE achète en moyenne $120 \mathrm{~kg}$ d'emballages chaque année. À l'échelle planétaire, cela correspond à 207 millions de tonnes équivalent à 384 milliards de dollars. Les emballages industriels et commerciaux représentent quant à eux près du double de ces volumes et de ces valeurs. Parallèlement, l'emballage apparaît comme la source de déchets la plus perçue par I'opinion publique, alors que concomitamment sa meilleure " circularité » permettrait de réduire ses coûts, de produire de la valeur sur l'ensemble du cycle de vie du produit et de créer des emplois via sa valorisation (réemploi, décomposition, recyclage, biodégradabilité, etc.) (World Economic Forum 2015). Quant à la fonctionnalité logistique d'un packaging, n'est-elle pas, à tort, trop largement considérée comme une opération isolée?
Dans le numéro d'octobre 2016 de Supply Chain Magazine, Karima Boukouna, Directrice des Opérations et du Service Client de Philips Health Systems France et membre du Comité Pack Experts œuvrant en amont du salon biennal de l'emballage All4pack qui s'est tenu au mois de novembre dernier à Paris, déclarait : "À la tête d'un centre de distribution européen de Philips Lighting, j'ai pu constater que l'emballage était encore trop souvent envisagé de manière isolée, sous le simple angle de la protection du produit ou du marketing, sans suffisamment prendre en compte ses implications logistiques [...]. Sans compter que c'est l'emballage qui porte les informations permettant à la supply chain d'être performante». De ce fait, son fort impact sur la supply chain plaide naturellement en faveur de son intégration selon une conception holistique (Sarkis 2003). Le packaging a effectivement une relation forte avec les autres composants du cycle de vie du produit (Sarkis 2003 ; 
Nilsson, Olsson et Wikström 2011 ; Woronoff 2014), d'où la notion de couple « produit-emballage » privilégiée par les spécialistes du packaging, et visible notamment à travers les problématiques de gaspillages alimentaires (liés en partie aux emballages inadéquats) ou celles de green supply chains.

Ainsi, le packaging remplit un grand nombre de fonctions, ${ }^{2}$ tant sur le plan de la qualité du produit, de la logistique que sur celui du marketing (Hellström et Saghir 2007 ; Verghese et Lewis 2007 ; Molina-Besh et Pålsson 2016). Parmi ces fonctions, bien que la plupart du temps sous-estimées voire ignorées par les consommateurs, les Fonctions Logistiques du Packaging (FLP) sont centrales. Ceci fait d'ailleurs dire à Fontaine (2016), Président du Conseil national de l'Emballage (CNE) et expert en innovation produits, propriété industrielle et développement durable, que le « citoyen consommateur " ne voit plus les emballages tant ils « sont cachés au service des produits qu'ils contiennent [...]. Quand le citoyen trie les emballages devenus vides, il n'imagine pas un seul instant le pourquoi et le comment de leur parcours ». Du côté académique ne peuton pas faire le même constat puisqu'à ce jour bien peu de littérature scientifique se consacre aux FLP ? Elle se limite bien souvent à étudier les trois principales contraintes mécaniques que doit satisfaire un emballage (manutention, stockage et transport), ou bien les trois contraintes climatiques (températures, humidité et rayonnement solaire).

Ce constat est encore plus marqué en ce qui concerne sa contribution à la construction d'une économie circulaire caractérisée par une circulation des flux physiques en "boucle fermée ", en réponse aux divers objectifs économiques, écologiques et sociaux / sociétaux formulés par les acteurs de la supply chain et les parties prenantes (McDonough et Braungart 2012 ; Le Moigne 2014 ; Fulconis, Paché et Reynaud 2016). Aussi, dans le prolongement des travaux académiques portant sur la reverse supply chain et sur les " chaînes logistiques en boucle fermée " ou "closed-loop supply chain »(Souza 2013 ; Govindan, Soleimani et Kannan 2015 ; Schenkel et al. 2015), des investigations sont-elles à mener sur les compétences logistiques-SCM qui pourraient renforcer la contribution des FLP à la transition vers une économie circulaire.

Dans ce contexte, cet article adopte une posture novatrice du packaging : analyser ses fonctions logistiques par rapport à leur capacité à favoriser la mise en œuvre d'une économie circulaire. Après avoir précisé le lien « emballage-logistique » dans une perspective d'économie circulaire, et ce, en considérant l'emballage comme un atout pour la performance de la logistique dans une approche circulaire des chaînes logistiques multi-acteurs (1), la notion traditionnelle de packaging scorecard est revisitée et un modèle de recherche est proposé (2). Il pose les bases d'une étude empirique visant à recueillir, sur cette problématique au cœur des préoccupations actuelles des chercheurs et des praticiens de la logistique, mais aussi des responsables politiques au plus haut niveau, ${ }^{3}$ les perceptions d'experts de l'industrie du packaging (3).

\section{Lien « emballage-logistique » dans une perspective d'économie circulaire}

Clarifier la contribution des FLP à la transition vers une économie circulaire conduit à inscrire la réflexion à la fois dans les contributions managériales et dans l'atteinte d'objectifs au niveau de la sphère politico-économique. À ce sujet, il ressort que les professionnels de l'emballage sont en première ligne et, en étant force de propositions, constituent des acteurs clés du passage d'une économie linéaire à une économie circulaire. Se pose alors la question du lien entre la logistique et l'emballage dans une perspective d'économie circulaire, comme celle des ressources et des compétences nécessaires pour promouvoir une telle transition, mais aussi celle des acteurs privés et publics concernés voire impliqués.

\section{D'une approche linéaire à une approche circulaire de l'économie, les acteurs de la chaîne de valeur de l'emballage en première ligne}

S'inscrivant dans le cadre du développement durable, l'économie circulaire est un modèle économique initié par une institution politique ou un gouvernement (" paradigme politique »), ancrée dans et agissant sur différents niveaux d'un territoire donné (local, régional, national ou international). L'économie circulaire vise ainsi à favoriser le développement économique d'un territoire donné, en privilégiant une circulation des flux physiques, d'eaux et d'énergie ainsi que des échanges en boucle fermée (Zhu, Geng et Lai 2010). Elle est un élément de l'écologie industrielle (Park, Sarkis et Wu 2010), imitant le fonctionnement des écosystèmes naturels caractérisés par des capacités d'organisation autonome et des mécanismes de retour d'information ou feed-back (Zhu, Geng et Lai 2010). Cette démarche se justifie par de moindres impacts négatifs tant sur l'environnement que sur le domaine social (BonetFernandez, Petit et Lancini 2014). Elle s'oppose à l'économie linéaire dont les modèles de production et de consommation, prévalant depuis la révolution industrielle, reposent sur l'exploitation des ressources naturelles supposées abondantes. ${ }^{4}$

Dans la littérature académique, le lien entre économie circulaire et logistique-SCM n'apparaît que partiellement. Les auteurs en Sciences de Gestion ont effectivement jusqu'alors préféré rapprocher la logistique et le SCM du développement durable et de la Responsabilité Sociale des Entreprises (RSE). Ceci s'observe tout particulièrement dans des problématiques portant sur des objectifs supposés antagonistes (économique, écologique et social), même si Massaroni, Cozzolino et Wankowicz 
(2014) considèrent la logistique en boucle fermée comme un outil indispensable à la réalisation du développement durable. Dans le même sens, Zhu, Sarkis et Lai (2008) postulent que les pratiques conformes au SCM durable contribuent à fermer les boucles logistiques. Bref, plusieurs concepts coexistent actuellement sans que leur intégration ni leur articulation n'aient été réalisées d'une manière convaincante. Pourtant, suite au rapport Brundtland (1987), les initiatives ne manquent pas. Bertolini (1995) soulignait le développement spectaculaire de l'emballage lié à l'évolution des modes de vie et de consommation, lequel s'accompagnait « de coûts croissants associés à l'après-usage, d'impacts environnementaux et de contestations en termes de gaspillage ». En attirant l'attention sur "la double vie de l'emballage ", Bertolini (1995) tentait de sensibiliser les acteurs économiques et politiques sur le fait qu'après la première vie de l'emballage centrée sur le couple « produit-emballage», arrive la seconde où la séparation marque "le passage de sa vie privée à sa vie publique, en tant que déchet, et elle pose le problème de son après-usage ».

Plus récemment, le Conseil National de l'Emballage $(\mathrm{CNE})^{5}$ publiait un rapport dans lequel est proposée une définition de l'économie circulaire dédiée aux emballages : "l'économie circulaire, pour le secteur de l'emballage, ne se limite pas au recyclage, elle couvre tous les stades de la vie du produit emballé à savoir : la conception, la production, la distribution et l'usage sans oublier la valorisation de l'emballage. Elle inclut les notions d'ancrage dans les territoires et de proximité » (CNE 2014). Dans cette vision résolument circulaire de la vie de l'emballage, ce rapport insiste également sur la place centrale qu'occupent les économies de ressources (matière, eau, énergie) rendues possibles par diverses démarches (éco-conception du couple " produit-emballage », réutilisation des emballages notamment en $B 2 B$, prévention des déchets d'emballage, amélioration de la recyclabilité, bouclage des flux de matériaux par réutilisation de la matière, etc.) (CNE 2014). Si ces professionnels de l'emballage semblent s'être pleinement appropriés la problématique de l'économie circulaire, ils font également preuve d'une grande perspicacité quant à son ancrage dans le paysage de l'emballage via leur dimension logistique.

Ainsi, le CNE a publié en 2015 un nouveau rapport consacré, quant à lui, à l'emballage comme acteur de la logistique des produits. II y est tout particulièrement mis en évidence que la logistique de tout couple « produit-emballage » doit se réfléchir " en pensant le système complet de l'emballage (emballage primaire, emballage secondaire et emballage de transport), en intégrant des stratégies d'économie circulaire (éco-conception, recyclabilité, reverse logistics, mutualisation des moyens entre acteurs, etc.), dans sa globalité car les choix d'entreprises qui sont réalisés à un endroit de la chaîne logistique peuvent impacter sur un autre endroit de la chaîne : le modèle économique choisi par un acteur de cette chaîne logistique n'est pas sans conséquence sur le modèle économique des autres acteurs »(CNE 2015). Comme l'illustre la Figure 1, « logistique, emballage et économie circulaire » constituent désormais trois notions difficilement dissociables à un moment où, plus que jamais, la volonté de réduire les coûts logistiques s'accentue, celle de réduire l'impact négatif de l'emballage sur l'environnement se confirme ${ }^{6}$ et l'amélioration de la performance sociétale des organisations (privés, publiques), notamment par la création d'emplois, est une priorité. $^{7}$

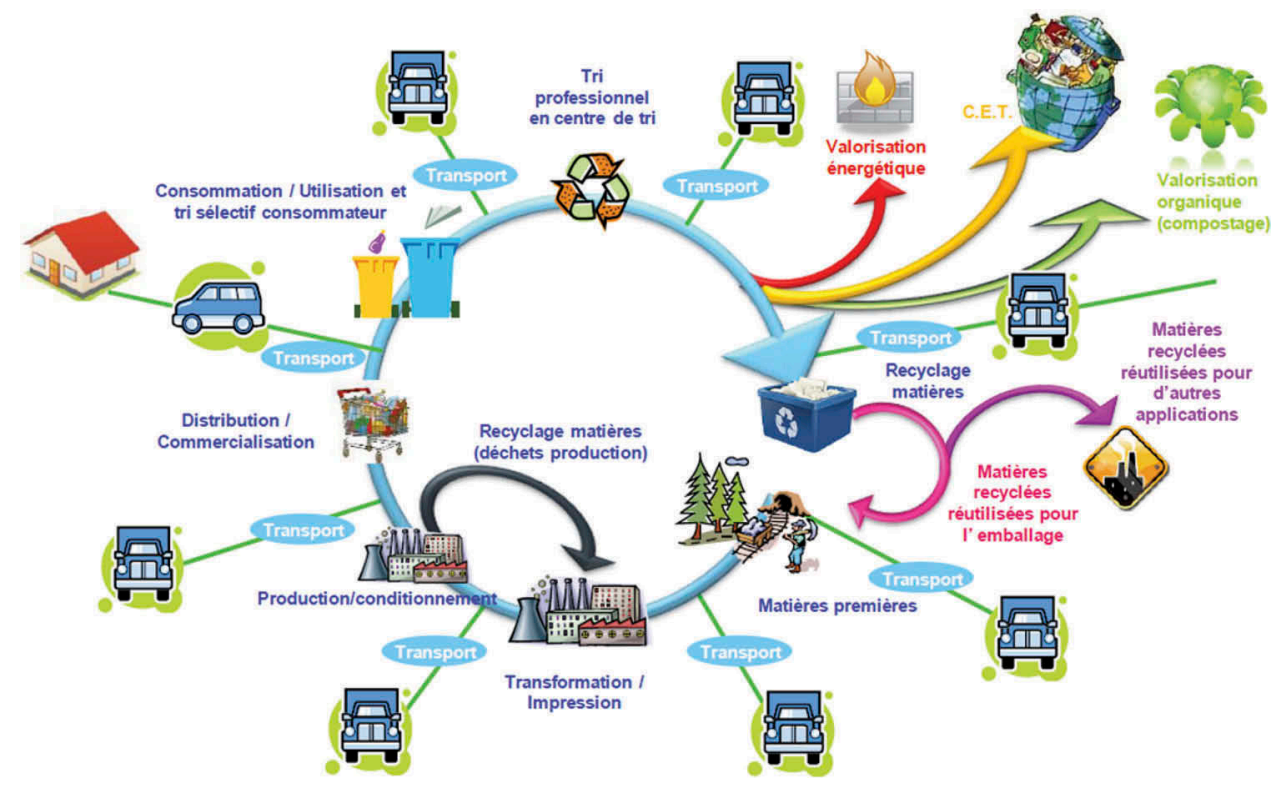

Figure 1. Logistique, emballage et économie circulaire : un trio indissociable.

Source : D'après CNE $(2015,8)$. 


\section{Lien entre logistique et emballage : intérêts d'une approche multi-acteurs}

Établir un lien entre logistique et emballage, dans une perspective d'économe circulaire, peut difficilement se limiter à I'acception stricto sensu des FLP, c'est-à-dire une acceptation cantonnée à des fonctions de transport, de manutention, de stockage auxquelles sont parfois ajoutées des fonctions de préhension et de rangement du produit pour le consommateur (Bertolini 1995; Devismes 2005). Parmi les tentatives d'élargissement des FLP qui émanent du périmètre de la logistique, on peut évoquer les travaux de Mentzer, Flint et Hult (2001) ou de Bienstock et al. (2008) qui permettent de définir la qualité de service logistique par les sept «b », à savoir : la bonne quantité, du bon produit, au bon endroit, au bon moment, dans les bonnes conditions, au bon prix, avec les bonnes informations. Cependant, selon ces conceptions des FLP liées au couple " produit-emballage ", les pratiques des acteurs de la chaîne logistique ne sont que partiellement prises en compte. II convient alors de replacer ces FLP dans un contexte plus large, notamment celui des chaînes logistiques multi-acteurs.

C'est ainsi qu'un groupe de travail du CNE a identifié en 2015 cinq thèmes établissant un lien entre logistique et emballage, considérant que " chaque acteur de la chaîne logistique intègre ces cinq thèmes dans sa stratégie d'entreprise : il doit trouver un équilibre entre eux et parfois réaliser des arbitrages tout en se conformant aux règles en vigueur " (CNE 2015). Selon cette conception, les FLP sont non seulement appréhendées à partir de leurs fonctions traditionnelles, mais aussi de ces cinq thèmes desquels elles relèvent (CNE 2015), à savoir :

- protection : il s'agit de la propriété de protection par l'emballage des produits (protéger le produit de ses propres caractéristiques en prolongeant sa durée de vie, protéger l'environnement extérieur du produit contenu, protéger le produit des contraintes extérieures, lutter contre le gaspillage et les pertes du produit, etc.) et des personnes (salariés, consommateurs, utilisateurs) notamment par le poids des produits emballés et des palettes, par la conception des linéaires, par les contraintes logistiques du passage en caisse, par les risques liés à la hausse des températures pendant le transport et le stockage, etc. ;

- environnement : ce thème se rapporte à la prise en compte de l'environnement dans la conception des emballages (démarche d'éco-conception) pour que ceux-ci contribuent à une logistique performante tout en respectant les fonctions attendues de l'emballage. Celui-ci est ici perçu comme un levier dans les démarches de RSE et de développement durable. Les principaux enjeux résident dans : la prévention par réduction à la source avec la question du couple " produitemballage », la réduction du transport entre fournisseur d'emballages et conditionneur, les gains logistiques en repensant le couple " produitemballage ", l'usage d'emballages industriels réutilisables et le recyclage des emballages ;

- économique : ce thème concerne l'excellence opérationnelle de la logistique (process, conditionnement en amont, stockage, préparation de commandes et distribution en aval) favorisée par l'emballage qui doit être adaptable (standardisation et différenciation) et multi-canal (distribution du produit et " mix-logistique »). Ainsi, l'emballage joue un rôle clé dans la mise à disposition du produit à l'utilisateur, depuis un site de production, de stockage, de cross-docking ou de distribution, voire lors de son transport par le consommateur ;

- information et communication : il est ici question de l'importance de l'emballage comme support d'informations et de communication tout au long de son parcours logistique. La fonction d'information est à la fois utile aux logisticiens (préparation de commandes, reconnaissance d'unité logistique), au personnel de magasin de distribution (mise en linéaire) et aux consommateurs (reconnaissance du produit, etc.). La fonction de communication est davantage utile aux opérations de merchandising, notamment à l'aide de l'emballage de transport (box, intercalaires, etc.), et de traçabilité où l'emballage est souvent un objet ou un support de traçabilité (traçabilité totale, information sur la recyclabilité) ;

- utilisation, usage, emploi : il est ici question du système complet de l'emballage (primaire, secondaire et tertiaire) tout au long du parcours logistique du couple " produit-emballage ». Les étapes lors desquelles l'emballage joue un rôle clé sont tour à tour la production et le conditionnement du produit, le transport en $B 2 B$ (multimodal, hygrométrie, etc.), le stockage et la préparation de commandes, le transport en $B 2 C$ et I'utilisation, le stockage et le tri par le client final, sans négliger les fonctions grandissantes de l'emballage dans l'e-commerce.

Certains travaux académiques sur ces thématiques rejoignent les réflexions du CNE (2015). À titre d'exemple, Massaroni, Cozzolino et Wankowicz (2014) avancent que le SCM durable a besoin de " packaging durable ». Par une étude exploratoire, ils mettent en évidence que le « réseau d'organisations » est une approche à privilégier pour appréhender les dimensions multi-secteurs, multiboucles, multi-métiers ainsi que les enchevêtrements verticaux comme horizontaux caractérisant les capacités et compétences que les acteurs des chaînes 
logistiques doivent mobiliser. Forts de ces constats, deux grandes questions de recherche émergent :

\section{QR1 : Quels sont les éléments des FLP susceptibles de favoriser ou de freiner la transition vers une économie circulaire?}

QR2 : Quelles compétences et capacités logistiquesSCM seraient à mobiliser ou bien à développer pour renforcer la performance des éléments des FLP tout en favorisant la transition vers une économie circulaire?

Bien qu'elle puisse paraître logique, l'articulation entre ces deux questions de recherche reste paradoxalement peu développée. Néanmoins, certains auteurs privilégient l'approche par les ressources pour étudier l'emballage (Jahre et Hatteland 2004). Se démarquant de l'approche le considérant comme une simple activité logistique, ces auteurs conçoivent l'emballage comme une ressource qui est connectée à d'autres ressources. Ainsi, l'emballage s'interface à de multiples autres ressources, telles que le produit, les infrastructures logistiques, mais aussi les acteurs, les compétences et les capacités logistiques-SCM associées.

\section{Nécessité d'un nouveau packaging scorecard}

Pour apporter des éléments de réponse aux deux questions de recherche formulées précédemment, des investigations ont été menées sur l'existence et la pertinence de packaging scorecards. Si des travaux existent, ils ne prennent pas en compte la problématique de l'économie circulaire. Un nouveau packaging scorecard est alors envisagé. II conduit à la proposition d'un modèle de recherche.

\section{Vers un packaging scorecard adapté à une conception circulaire de l'économie}

Depuis presque trois décennies, nombreuses sont les tentatives d'élaboration d'outils pour mesurer les activités d'une entreprise. Le tableau de bord prospectif, plus connu sous le vocable de Balanced Scorecard, proposé par Kaplan et Norton $(1992,1996)$ en est l'exemple le plus marquant. II fut décliné dans divers domaines tels que les approvisionnements (Pratsch et Ustad 1996) ou les emballages et la logistique (Johnsson 1998). Au-delà d'un simple instrument de mesure, ce sont ses intérêts en termes de formulation de la stratégie des entreprises et des réorganisations qui s'ensuivent selon une approche holistique qui ont inspiré Olsmats et Dominic (2003). Convaincus de la nécessité de privilégier une telle approche du packaging, et face à l'allongement et la complexité des chaînes logistiques, ces deux auteurs ont alors proposé un tableau de bord spécifique au packaging, le « packaging scorecard» (Olsmats et Dominic 2003). L'originalité de ce tableau de bord réside dans le fait qu'il prend en compte les différents acteurs des chaînes logistiques. Pour chaque acteur et dans le but d'accroître sa performance, une évaluation du rôle et des fonctions du packaging est effectuée. Ce packaging scorecard vise également à atteindre l'objectif commun de l'ensemble des acteurs de la chaîne logistique, la satisfaction du client final.

Fondée sur la recherche de critères fonctionnels du packaging, c'est donc une approche davantage holistique de la contribution du packaging à la création de valeur tout au long des chaînes logistiques qui est développée par Olsmats et Dominic (2003). Dans le même esprit, des initiatives provenant d'entreprises privées sont également à noter comme l'atteste la démarche initiée, en 2006, par le géant de la distribution Walmart. Cette année-là, Walmart dévoila son propre packaging scorecard à destination de ses fournisseurs, ${ }^{8}$ packaging scorecard qui se voulait un outil de mesure leur permettant de s'évaluer par rapport à d'autres fournisseurs dans le but de réduire les emballages (matériaux, poids) tout en maintenant leurs propriétés en termes logistiques. Cependant, les initiatives que l'on peut recenser à ce jour, toutes originales qu'elles soient en privilégiant une approche multiacteurs, sont peu nombreuses et se limitent le plus souvent à une approche linéaire des chaînes logistiques. Le packaging scorecard proposé par Olsmats et Dominic (2003) s'appuie en effet sur un découpage assez réducteur des chaînes logistiques et ne prend en compte que quatre catégories d'acteurs, à savoir : les fournisseurs, les transporteurs et les grossistes, les détaillants et, enfin, les consommateurs.

Or, dans une conception circulaire de l'économie, la prise en compte de nouveaux acteurs ne devientelles pas incontournable ? En effet, au-delà de l'entreprise, industrielle ou de distribution, ne faut-il pas élargir l'analyse aux rôles joués par les acteurs de la reverse supply chain, comme à ceux joués par le consommateur (qui ne serait plus le dernier maillon de la chaîne logistique mais un maillon à part entière dans une chaîne logistique en boucle fermée), par des écoorganismes, des associations, des scientifiques et, plus largement, par les pouvoirs publics qui sont partie prenante, $^{9}$ voire le moteur, de la transition vers l'économie circulaire ? À l'évidence, un packaging scorecard adapté à une conception circulaire de l'économie s'impose. Outre les critères traditionnels du packaging qui sont généralement les plus importants pour les différents acteurs de la chaîne logistique et utilisés par Olsmats et Dominic (2003) (facilité de fabrication, protection du produit, flux d'information, efficacité poids et volume, qualité et taille, maniabilité, capacité de vente, sécurité, réduction de l'utilisation des ressources, utilisation minimale de 
substances dangereuses, quantité minimale de déchets, coûts d'emballage, autres propriétés à valeur ajoutée), d'autres critères fonctionnels du packaging relevant de dimensions plus englobantes ou de nouvelles dimensions sont à prendre en compte en fonction des acteurs concernés. Ainsi, contrairement au packaging scorecard traditionnel qui a pour objectif la satisfaction du client final, le nouveau packaging scorecard se doit de considérer également d'autres objectifs prenant en compte le lien entre économie circulaire et sphère politico-économique.

\section{Proposition d'un modèle de recherche}

Poser les bases d'un nouveau packaging scorecard délibérément orienté vers l'économie circulaire fait apparaître deux catégories de critères : d'une part les traditionnels pouvant être appréciés quantitativement mais qui restent à resituer en fonction de nouveaux thèmes dont ils relèvent et, d'autre part, de nouveaux se référant à des compétences et à des capacités logistiques-SCM susceptibles de renforcer la performance des éléments des FLP tout en favorisant la transition vers une économie circulaire. Nous sommes donc face ici à deux niveaux d'analyse qui font écho aux deux questions de recherche que nous avons préalablement formulées.

Selon la première question de recherche $(Q R 1)$, il paraît nécessaire de s'interroger sur les FLP favorisant ou freinant la transition vers une économie circulaire. Le nouveau packaging scorecard reprend du modèle initial le poids respectif de chaque critère FLP ainsi que le résultat ou le score obtenu. L'orientation vers l'économie circulaire se manifeste par l'intégration, en plus de la boucle emballage, des boucles plus larges vers d'autres utilisations (CNE 2015), la considération des divers acteurs ou parties prenantes dépassant des logiques traditionnelles $B 2 B$ et $B 2 C$, ainsi que la prise en compte des tous les systèmes d'emballages (primaire, secondaire et tertiaire). De la même façon et conformément à la définition de l'économie circulaire, le nouveau packaging scorecard s'applique aux problématiques multi-cycles, multi-maillons, multiacteurs et multi-secteurs. II s'agit d'un concept holistique susceptible d'être adapté à un contexte spécifique (exemple : délimitation du territoire d'intervention) pour fournir des résultats pertinents.

Selon la seconde question de recherche (QR2), une fois les éléments des FLP et leur poids respectif identifiés, il s'agit de mobiliser en interne ou en externe des compétences et des capacités logistiques-SCM afin d'obtenir un niveau de performance souhaité pour les FLP. Ces compétences et capacités interviennent souvent à des niveaux différents par rapport à la définition des FLP. En effet, I'identification des FLP se situe au niveau du design / développement / innovation du couple « produit-emballage » autrement dit en amont, tout comme les compétences et les capacités logistiques-SCM. Toutefois, ces dernières sont également mobilisées lors de la réalisation des performances relatives aux FLP qui renvoie à des dimensions plus tactiques ou opérationnelles. D'une manière générale, les compétences et les capacités apparaissent comme des concepts clés dans plusieurs théories du management stratégique (Prévot, Brulhart et Guieu 2010), dont la théorie des ressources, la perspective fondée sur le management stratégique des compétences, la théorie fondée sur les connaissances, les capacités dynamiques et l'approche relationnelle. Bien que reliées et complémentaires (Prévot, Brulhart et Guieu 2010), ces cinq perspectives présentent des spécificités et des nuances, dont l'agencement adéquat paraît tout particulièrement prometteur pour des problématiques en SCM généralement de nature dynamique, relationnelle et collaborative.

Si les compétences et les capacités logistiques pertinentes pour le SCM ont été identifiées par la communauté scientifique (Duong et Paché 2015), c'est généralement sans référence explicite au packaging ou à l'économie circulaire. Ainsi, Gammelgaard et Larson (2001) distinguent entre capacités générales et compétences, ces dernières étant uniques et liées à un contexte bien précis. Ces auteurs trouvent empiriquement les capacités et les compétences les plus importantes en SCM, en opposant enquêtes quantitatives selon une double perception professionnels / étudiants, et recherche qualitative via des études de cas. Cette triangulation méthodologique permet de dresser un inventaire assez exhaustif, les éléments mesurés concernant les compétences et les capacités techniques, informatiques, statistiques, générales / relatives au management, intra-firme comme interfirmes, systémiques-holistiques (" the big picture »), communicatives, éthiques et humaines (l'empathie et l'écoute).

Les capacités renvoient donc au double niveau intra-entreprise et inter-entreprises, ces dernières ayant une portée clairement stratégique, parce qu'elles sont socialement complexes, causalement ambigües et historiquement évolutives (Gold, Seuring et Beske 2010), autrement dit le fruit d'interactions entre acteurs. Ce paradigme collaboratif caractérisant les chaînes logistiques considère effectivement la collaboration stratégique comme source de compétitivité. II s'étend désormais également à des aspects écologiques et sociétaux (Vachon et Klassen 2006), tout particulièrement aux problématiques situées à l'interface SCM / économie circulaire. Concernant les compétences logistiques-SCM au service de la performance des éléments des FLP dédiées à l'économie circulaire, aucune validation empirique n'a été réalisée à ce jour, même si les chercheurs s'accordent sur l'importance des capacités inter- 


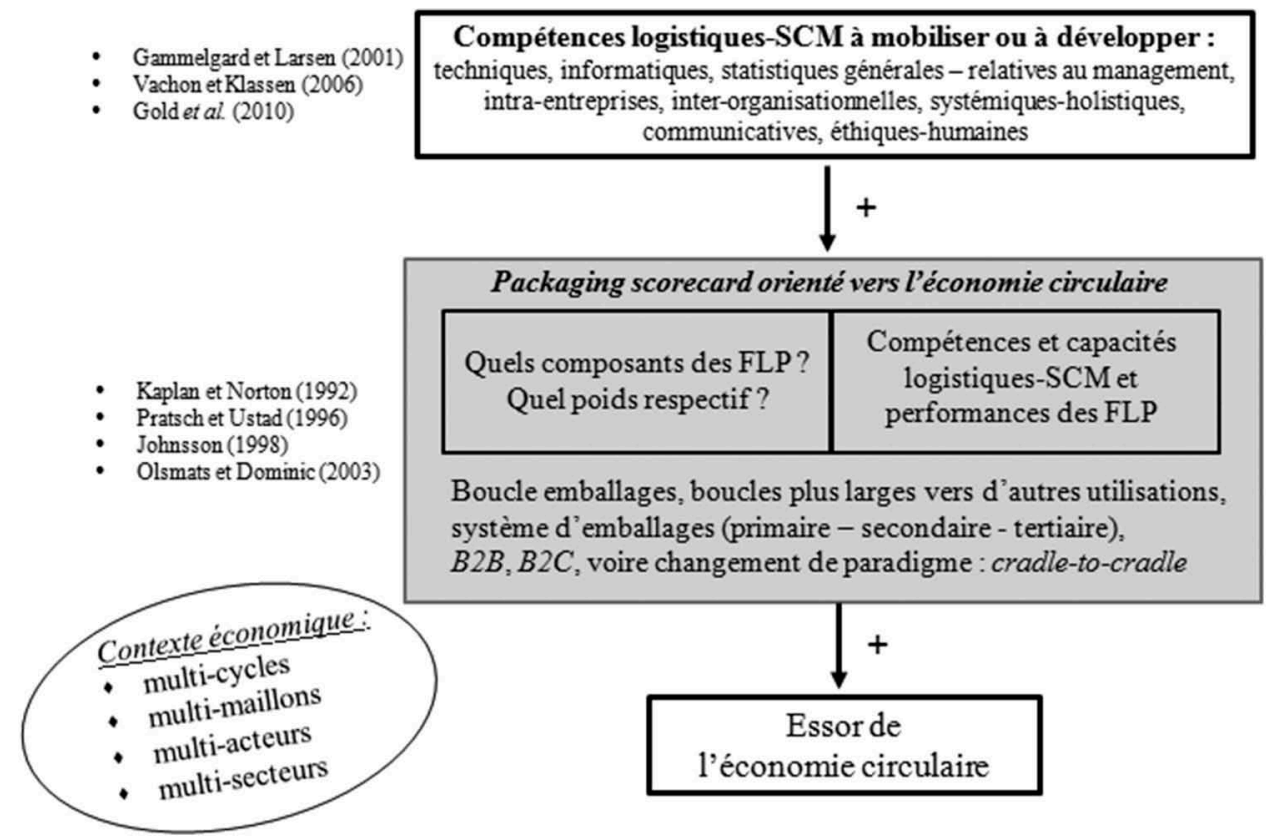

Figure 2. Modèle de recherche pour un packaging scorecard adapté à une vision circulaire de l'économie.

Source : Élaboration personnelle.

entreprises, localisées auprès de quasiment tous les acteurs (traditionnels, nouveaux, privés comme publics) de la chaîne logistique.

Face à ce constat, nous proposons (cf. Figure 2) un modèle de recherche relatif à un packaging scorecard adapté à une conception circulaire de l'économie.

\section{Emballage et économie circulaire, la perception d'experts de l'industrie du packaging}

Afin d'analyser les FLP par rapport à leur capacité à favoriser la mise en œuvre d'une économie circulaire, nous avons souhaité recueillir les perceptions d'experts de l'industrie du packaging. Après avoir précisé le dispositif de recueil des données empiriques, les premiers résultats de cette consultation d'experts sont présentés et discutés, notamment en vue de recherches à venir à réaliser à plus grande échelle.

\section{Dispositif de recueil des données empiriques}

C'est à l'aide d'un questionnaire qu'a été testée la pertinence de notre modèle de recherche relatif à un packaging scorecard adapté à une conception circulaire de l'économie et qu'ont été recherchés des éléments de réponse aux deux questions de recherche. Les items du questionnaire associent les apports théoriques du packaging scorecard traditionnel d'Olsmats et Dominic (2003) aux cinq thèmes identifiés par le CNE (2015). Des éléments inédits provenant notamment du domaine social ont également été pris en compte. En effet, ceux-ci ont longuement été négligés par les chercheurs comme par les professionnels, alors qu'ils sont un composant essentiel de l'économie circulaire. Pour élaborer les items du questionnaire, l'absence d'un cadre théorique complet adapté à notre problématique nous a amenés à associer des sources scientifiques à des sources réglementaires et des publications pseudo-scientifiques (type think tank). Le questionnaire se compose de sept grandes parties:

- tout d'abord, dans une perspective de mise en œuvre de l'économie circulaire, le questionnaire mesure 41 éléments FLP. Cette mesure s'effectue, d'une part, par l'importance que leur attribue de façon générale le répondant et, d'autre part, par la performance effective perçue par celui-ci par rapport à sa dernière expérience concrète avec un emballage (primaire, secondaire ou tertiaire) ;

- il comprend ensuite deux questions ouvertes permettant au répondant d'ajouter des commentaires (nature des construits sur les FLP), ou bien d'observer des antagonismes entre certains " couples » FLP susceptibles de freiner l'essor de l'économie circulaire ;

- il s'ensuit un groupe de 11 questions qui renvoie aux compétences et aux capacités logistiquesSCM des acteurs susceptibles de favoriser l'essor de l'économie circulaire. Comme pour les 41 éléments $F L P$, nous interprétons les compétences comme des combinaisons uniques de ressources (Hamel et Prahalad 1990) pouvant impacter positivement l'évolution vers l'économie circulaire. Mais, contrairement aux éléments FLP, la mesure relative aux 
compétences ne concerne que l'importance attribuée (faible à forte) et non le score réel, ce dernier échappant au périmètre d'observation du répondant qui n'a généralement pas une visibilité complète sur les compétences déployées ou à déployer à tous les niveaux de la chaîne logistique ;

- une question ouverte sur ces compétences et capacités complète ce groupe de questions ;

- un groupe de quatre questions permet ensuite au répondant de donner sa perception de l'économie circulaire en 2016, en particulier sur sa maturité ;

- sur cette même thématique, une question ouverte est alors proposée permettant au répondant de donner un avis complémentaire sur les caractéristiques de l'économie circulaire ;

- le questionnaire se termine enfin par une partie signalétique classique.

La structure du questionnaire, qui tient compte des améliorations apportées suite à la phase de pré-test, est résumée dans le Tableau 1 ci-dessus.

Une trentaine d'experts de l'industrie du packaging, adhérents au CNE, constitue le public cible définitif du questionnaire. II s'agit tout d'abord de représentants du CLIFE, le Comité de Liaison des Industries Françaises de l'Emballage qui regroupe différents experts d'organismes spécialisés dans le verre (CSVMF-VERRE AVENIR), les emballages plastiques et souples (ELIPSO), le cartonnage et les articles de papeterie (FFCP), la production du carton ondulé (Carton Ondulé de France), le papier et le carton d'emballage (REVIPAC), les emballages industriels et la logistique associée (SEILA), les emballages légers en bois (SIEL), les emballages métalliques (SNFBM) et les palettes en bois (SYPAL).

On retrouve également tour à tour des experts représentant : ECO-EMBALLAGES, l'éco-organisme qui a pour mission d'organiser le dispositif national de tri et de recyclage des emballages ménagers pour réduire leur impact sur l'environnement ; la FÉDÉRATION DE LA PLASTURGIE ET DES COMPOSITES, organisation représentative des entreprises de la transformation des matières plastiques ; le GEPPIA, Groupement des Équipementiers du Process et du Packaging des Industries Agroalimentaires et non-alimentaires; le SECIMEP, Syndicat des Entreprises de Commerce International de Matériels d'Emballage et de Process ; et INTEREMBALLAGE SA (ex-Interfilières) qui regroupe les cinq filières de matériaux d'emballage au sein d'EcoEmballages (pour le secteur plastique Valorplast, pour le secteur aluminium France Aluminium Recyclage, pour le secteur acier ArcelorMittal Packaging, pour le papier carton Revipac, et pour le secteur verre la Chambre Syndicale des Verreries Mécaniques de France - CSVMF).

Figurent aussi des représentants de la FCD, Fédération des Entreprises du Commerce et de la Distribution ; de la FÉDÉRATION FAMILLES DE FRANCE, organisation nationale de consommateurs qui assure la défense des consommateurs ; de I'ILEC, Institut de Liaisons et d'Études des Industries de Consommation ; de I'INDP, Institut National du Design Packaging ; du LNE, Laboratoire National de métrologie et d'Essais ; de PIK PIK Environnement, association loi 1901, d'intérêt général, qui a pour but l'éducation à l'éco-citoyenneté et à l'environnement urbain ; et, enfin, l'AMF, l'association des maires de France.

Quant au questionnaire, avant son administration finale, il a été pré-testé en deux temps auprès de huit experts en packaging (printemps 2016). Dans un premier temps, quatre experts en packaging ont commenté le questionnaire sans le remplir entièrement (intitulés P1, P2, P3 et P4), il s'agissait de deux enseignantschercheurs en marketing, un enseignant-chercheur en SCM ainsi qu'un professionnel en SCM, tous familiarisés par leur métier avec le monde du packaging. Leurs retours ont abouti à une première amélioration du questionnaire initial. Ainsi, les pourcentages du packaging scorecard initial, utilisés par Olsmats et Dominic

Tableau 1. Structure du « questionnaire experts » sur l'importance des FLP face à l'essor de l'économie circulaire.

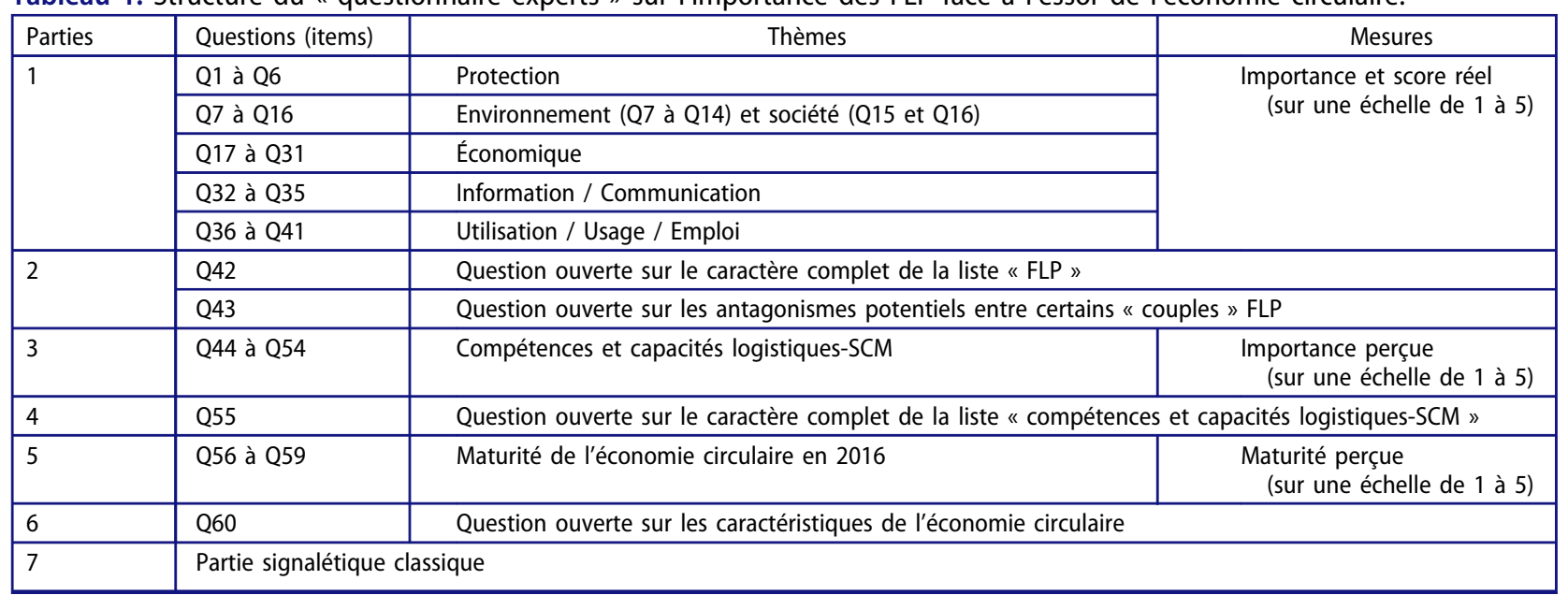

Note : Le questionnaire est disponible sur simple demande auprès des auteurs. 
(2003) pour mesurer l'importance attribuée de façon générale à chacun des 41 éléments FLP identifiés (colonne 1 du questionnaire), ont été transformés en échelle de Likert (avec cinq choix de réponse) afin d'éviter des malentendus (la somme des importances relatives aux éléments FLP dépasse généralement $100 \%$, mais tous les répondants n'auront pas la même interprétation). Une échelle à cinq points permet d'harmoniser la lecture, le score réel (colonne 2 du questionnaire) se mesurant également sur un intervalle de cinq points. Cette déviance du modèle initial, déjà considérée dans la Figure 2 et le Tableau 1 (cf. supra), se justifie dans la mesure où la perte de précision est négligeable.

Dans un second temps (mai et juin 2016) et considérant les premières améliorations, quatre professionnels « seniors » du packaging ont entièrement rempli le questionnaire (intitulés E1, E2, E3 et E4). Les améliorations de notre appareil de mesure pour une problématique inédite articulant FLP, économie circulaire et compétences logistiques-SCM font partie de nos résultats développés ci-après. Notons que durant la phase de pré-test, le questionnaire a été envoyé par voie électronique. Les quatre retours développés ciaprès (E1 à E4) associent des commentaires reçus par email avec des conversations téléphoniques (« administration assistée » du questionnaire). Entièrement remplis, ces quatre questionnaires permettent à ce stade une première exploitation plus qualitative que quantitative des données empiriques.

\section{Premiers résultats de la consultation d'experts : apports de la phase de pré-test}

Suite à la phase de pré-test reposant sur les quatre retours évoqués précédemment (notés E1, E2, E3 et E4), deux catégories de résultats émergent. Les premiers, de nature méthodologique, permettent de poursuivre l'amélioration du questionnaire amorcée par l'interrogation d'experts en packaging (P1 à P4, printemps 2016). Les seconds concernent directement les FLP, tant sur leurs aspects antagonistes que sur l'approche holistique privilégiée dans cette recherche. Ainsi, des premiers résultats, de nature qualitative, se rapportent à la contribution des compétences et des capacités logistiques-SCM à l'essor de l'économie circulaire.

Amélioration de l'appareil de mesure - Durant la phase de pré-test du questionnaire, les répondants ont souhaité des explications complémentaires pour bien comprendre la différence entre l'importance attribuée de façon générale à chaque élément FLP et la performance effective perçue de chaque élément FLP par rapport à une expérience concrète avec un emballage. La décision a alors été prise d'administrer le questionnaire "par téléphone » et, en fonction des réponses obtenues, de modifier à nouveau le packaging scorecard que nous proposons inspiré de celui d'Olsmats et Dominic (2003). À titre d'exemple, la colonne 2 relative au score réel, au lieu de s'appuyer sur une échelle de mesure à cinq points, pourrait reposer sur seulement trois appréciations : inférieure, égale et supérieure. À ce jour, les écarts positifs enregistrés (28 items sur 41 ) comme négatifs ( 9 items sur 41 ) entre importance moyenne et score réel moyen sont relativement limités, à savoir 1,75 et $-0,75$ respectivement sur l'échelle proposant des valeurs entre 1 à 5 . Parallèlement, 4 items affichent une moyenne identique pour l'importance et le score réel.

La phase de pré-test a également permis d'apporter des améliorations quant à la compréhension de certaines questions pouvant porter à confusion ou, par manque de précisions, empêchaient le répondant de se prononcer. En ce sens, un expert packaging (E2) conseille, à l'image de la question Q7 portant sur l'emploi de ressources et d'énergie à la source, d'ajouter une distinction entre matières " renouvelables " et " non-renouvelables ", déterminant selon lui la réponse à la fois pour l'importance et le score réel de chaque élément FLP. Dans le même esprit, le répondant E1 recommande de scinder (Q13) les éléments « matériaux » et " écodesign » d'un type particulier d'emballage pouvant faciliter le développement durable / la RSE. II s'agit en effet de deux éléments distincts à mesurer. Cela conduit à réfléchir sur la complexité méthodologique liée au dilemme suivant :

- d'un côté, l'importance de chaque élément FLP et peut-être plus encore de leur score variant certainement en fonction du niveau d'emballage (primaire, secondaire, tertiaire) et du type d'emballage considéré (alimentaire, automobile, mobilier, articles de sport, etc.) ;

- de l'autre, l'intégration de l'économie circulaire dans notre problématique nécessite une démarche dépassant la logique traditionnelle mono-produit ou mono-secteur.

Se limiter à un seul niveau et à un seul type d'emballage augmenterait, il est vrai, la précision relative à l'importance de chaque élément FLP et à son score réel. Cependant, cela réduirait le nombre de répondants potentiels, éventuellement non-familiarisés avec un emballage précis (suite à l'hétérogénéité des expertises packaging). Or, nous cherchons à mesurer la perception des répondants en tant qu'experts packaging et non pas en tant que simples consommateurs. Restreindre le périmètre des emballages considérés négligerait également la nature multi-secteur et multi-cycle sousjacente à l'économie circulaire. Le score réel renvoie à une expérience concrète avec un emballage à l'image des enquêtes de satisfaction en marketing qui se fondent sur un vécu effectif ( $\mathrm{E} 4$ précise qu'il se réfère dans ses 
réponses à une boîte en carton ouverte pour pommes biologiques, recouverte d'un film plastique). Se limiter à un même type ou niveau d'emballage signifierait s'adresser à des experts packaging du même maillon ou secteur (exemple : « LE » spécialiste de l'emballage souple) ce qui est contraire à la notion de l'économie circulaire. Pour pouvoir pousser plus loin ce raisonnement, l'articulation des trois éléments constitutifs de notre problématique (FLP, capacités et compétences logistiques-SCM et économie circulaire) semble créer des challenges inédits (?) - aux méthodologies traditionnelles. S'ouvre alors le débat les opposant aux méthodologies plus qualitatives telles que l'observation, l'expérience ou bien l'étude de cas (Jahre et Hatteland 2004), mais au prix d'échantillons moins importants et d'un potentiel réduit de généralisation.

FLP : entre antagonismes, modélisation holistique et compétences logistiques-SCM - Loin des synergies recherchées par les défenseurs de la transition vers une économie circulaire, les premiers retours des répondants mettent en exergue des conflits d'intérêts entre FLP, que ce soit à l'intérieur d'un même thème ou entre les cinq thèmes desquels elles relèvent. Ainsi, un professionnel (E1) souligne un antagonisme à l'intérieur du thème "Environnement et société ", plus précisément au sujet de l'éco-conception. Selon lui, la réduction de la quantité de matière nécessaire pour fabriquer un emballage et, de ce fait, de la quantité de déchets générée après son utilisation (Q9), rentre en conflit avec les questions de leur recyclabilité (Q7, Q12, Q13). En effet, dans une optique marketing et voulant casser les codes du marché en proposant un emballage souple, comment une entreprise peut-elle concilier la réduction du poids d'un emballage $100 \%$ recyclable avec l'ajout, nécessaire pour des questions de stabilité, de nouveaux matériaux empêchant alors tout recyclage?

Entre les cinq thèmes identifiés par le CNE (2015) et établissant un lien entre logistique et emballage, un autre professionnel (E2) dénonce des antagonismes entre celui de « Protection » et celui d'« Environnement et société », en particulier entre les sous-thèmes «Propreté / hygiène " et "Réutilisation ». Ce même professionnel (E2) mentionne un autre type d'antagonisme entre le thème "Environnement et société " et deux thèmes plus classiques associés aux FLP : «Économique » et "Information / communication ", plus précisément entre les questions du recyclage (Q9, Q12, Q13) et la qualité et la quantité du produit à recycler (Q27, Q38, Q39, Q40, Q41). Si l'information et la communication sur le produit (Q35) sont un élément FLP important, le packaging n'est-il pas physiquement surdimensionné ? Parallèlement, cela révèle un autre antagonisme entre les trois thèmes « Environnement et société » (Q9, Q10, $\mathrm{Q} 12, \mathrm{Q} 13)$, «Économique » (Q30) et « Utilisation / usage / emploi »(Q37).
Face à ces antagonismes, un des professionnels interrogés (E1) se questionne sur la contribution même des FLP à l'essor de l'économie circulaire (concept revendiquant les "boucles de matière "selon ses propos). Ce retour qualitatif pourrait conduire à remettre en question l'approche holistique retenue des FLP ou, plus généralement, de la logistique (triple bottom line), alors que simultanément l'objectif de la Proposition de directive du Parlement européen et du Conseil (2015 / 0276), relative aux emballages et aux déchets d'emballages, consiste justement à " promouvoir une économie plus circulaire » selon une telle approche. Notons par ailleurs que ces antagonismes rappellent ceux décrits par Nilsson, Olsson et Wikström (2011) et Wever (2011) dans le domaine spécifique du packaging, à travers des conflits entre fonctions logistiques, marketing et environnementales.

Les retours obtenus ne se limitent pas aux seules FLP, ils portent également sur la contribution des compétences et des capacités logistiques-SCM à l'essor de l'économie circulaire. Selon un expert packaging (E1) interrogé, " les fabricants d'emballages deviennent actuellement des recycleurs et des utilisateurs d'emballages ». Cela révèle deux tendances. Primo, les fabricants d'emballages deviendraient les " maîtres incontestés des processus de recyclage", plus précisément de leur efficience et de leur efficacité. Dès lors, ces acteurs-là ne seraient-ils pas les plus à mêmes de disposer des compétences et des capacités logistiques-SCM susceptibles de favoriser l'essor de l'économie circulaire ? Secundo, ce propos traduit également une concentration économique des secteurs et donc une forme supplémentaire de source de pouvoir, s'ajoutant à la légitimité visible à travers leurs compétences et leurs capacités. Ces retours qualitatifs sur la question (Q55) portant sur les compétences et les capacités des acteurs, et plus largement des parties prenantes, permettront de compléter l'évaluation quantitative des éléments constitutifs de ces compétences et capacités des acteurs (Q44 à Q54) susceptibles de renforcer la performance des FLP dans ce contexte.

\section{Conclusion}

Analyser les Fonctions Logistiques du Packaging (FLP) par rapport à leur capacité à favoriser la mise en œuvre d'une économie circulaire ne peut se satisfaire d'une approche purement technique de l'emballage. Si par nature le packaging concerne de nombreux professionnels (fournisseurs de matières premières, industriels, concepteurs, fabricants d'emballage, photograveurs / imprimeurs, transporteurs, distributeurs), abordé sous l'angle de l'économie circulaire, il convient non seulement d'élargir l'analyse du côté des consommateurs dont le rôle est de plus en plus important (ne devient-il pas le point de départ des 
reverse supply chains ?), mais aussi du côté d'autres acteurs, tels que les pouvoirs publics qui auront certainement une fonction de communication à tenir dans les prochaines années. Ne deviennent-ils pas de véritables parties prenantes au sens de Freeman (1984) dans le fonctionnement des chaînes logistiques en boucle fermée ? Dès lors, la question des compétences et des capacités logistiques-SCM des acteurs se pose incontestablement.

C'est ce que nous avons mis en évidence à travers la formulation de deux questions de recherche issues de la littérature, mais aussi par la nécessité d'élaborer un nouveau packaging scorecard adapté à une conception circulaire de l'économie, par la proposition d'un modèle de recherche et, enfin, par la présentation des premiers résultats issus d'un questionnaire pré-testé auprès d'experts de l'industrie du packaging. La phase de pré-test du questionnaire, organisée en deux tours (commentaires préliminaires suivis de questionnaires entièrement remplis via une administration assistée), a permis d'obtenir un appareil de mesure opérationnel. Désormais, son administration à grande échelle vise à apporter des éléments plus précis de réponse aux deux questions de recherche et de tester des hypothèses reliant les ressources (FLP et compétences logistiques-SCM) à l'essor de l'économie circulaire. Un échantillon composé de plus d'une trentaine d'experts du packaging dans la zone d'influence du CNE est à ce jour sollicité.

\section{Notes}

1. Les auteurs remercient très sincèrement les évaluateurs anonymes qui, par leurs commentaires, ont permis d'améliorer significativement une première version de l'article. Ils remercient également les répondants qui ont permis de réaliser une première consultation d'experts et d'améliorer sensiblement l'appareil de mesure.

2. Notons que la littérature, y compris scientifique, n'est pas toujours univoque concernant le terme packaging, notamment à propos de ses "fonctions " ou "rôles " (Jahre et Hatteland 2004), « exigences » (Rundh 2005), " besoins » (Molina-Besch et Pålsson 2016) et « impératifs » qu'elle utilise parfois comme synonymes. Il en est de même pour les notions de " design / redesign » (García-Arca et Prado Prado 2008), " développement » (Simms et Trott 2010), " solution » (Hellström et Saghir 2007), " choix / sélection » (MolinaBesch et Pålsson 2016) et "innovation 》 (Jahre et Hatteland 2004).

3. Cf. la « Proposition de directive du Parlement européen et du Conseil modifiant la directive 94 / 62 / CE relative aux emballages et aux déchets d'emballages » (2015 / 0276 COD). Elle concerne plus spécialement le " train de mesures sur l'économie circulaire qui englobe également une communication de la Commission intitulée 'Boucler la boucle - Un plan d'action de l'Union européenne en faveur de l'économie circulaire' ».

4. Informations tirées du site Internet : http://www.insti tut-economie-circulaire.fr (consulté le 31 mai 2016).
5. "Le Conseil National de l'Emballage (CNE), association créée en 1997, est une autorité morale réunissant les différents acteurs de la chaîne de valeur de l'emballage: producteurs de matériaux d'emballage, fabricants d'emballage, entreprises de produits de grande consommation, entreprises de la distribution, sociétés agréées et opérateurs du secteur de la collecte et de la valorisation, collectivité locales, associations de consommateurs et de protection de l'environnement, designers, fabricants de machines et autres professionnels de l'emballage » (d'après le site Internet suivant : http://www.conseil-emballage.org, consulté le 4 octobre 2016).

6. Selon le Rapport d'activité Éco-emballages 2012 (disponible sur : http://www.ecoemballages.fr), les emballages ménagers représentaient en France 4,5 millions de tonnes (67\% furent recyclés) et les déchets d'emballages industriels et commerciaux (DEIC) représentaient 7,5 millions de tonnes (70 \% furent recyclés).

7. Selon I'ASLOG, «écosystème majeur de notre économie », le secteur de la logistique regroupe entre 1,6 et 2 millions d'emplois en France parmi les acteurs de l'industrie, du négoce, de la distribution, des prestations logistiques et du transport et parmi les activités associées. Site Internet : http://www.aslog.org (consulté le 12 novembre 2016).

8. D'après le site Internet suivant : http://corporate.wal mart.com/_news_/news-archive/2006/11/01/wal-martunveils-packaging-scorecard-to-suppliers (consulté le 31 mai 2016).

9. En témoigne, par exemple, le protocole d'accord sur le barème de calcul des soutiens versés aux collectivités locales qui collectent et trient les déchets d'emballages ménagers signé en juillet 2016 entre I'Association des maires de France et des présidents d'intercommunalité, I'Association nationale des industries alimentaires (ANIA), la Fédération des entreprises de la beauté (FEBEA), la Fédération du commerce et de la distribution (FCD) et l'Association technique du commerce et de la distribution (Perifem) (http://www.economiecircu laire.org) (consulté le 12 novembre 2016).

\section{Déclaration}

Les auteurs confirment qu'il n'y a aucun conflit d'intérêt.

\section{Les auteurs}

François Fulconis est maître de conférences hors classe en Sciences de Gestion à l'Université d'Avignon et des Pays de Vaucluse (UAPV). Ancien du CEFAG (FNEGE-Paris), il est membre permanent du Centre de Recherche sur le Transport et la Logistique (CRET-LOG, Aix-Marseille Université) et membre associé au laboratoire LBNC (UAPV). Ses recherches s'inscrivent dans le domaine du management stratégique et logistique des organisations. Elles traitent majoritairement du management des structures en réseau, des stratégies de collaboration au sein des chaînes logistiques multi-acteurs et de l'intermédiation logistique, notamment à travers le rôle des prestataires de services logistiques (PSL). Ces thèmes ont fait l'objet de près de 150 publications (revues, ouvrage, chapitres d'ouvrages et colloques) nationales et internationales. II est par ailleurs membre du bureau de l'AIRL-SCM. 
Bernd Philipp est enseignant-chercheur en logistique et SCM à I'ESCE (Paris). Depuis son doctorat en Sciences de Gestion, et en tant que membre permanent du laboratoire CRET-LOG (Aix-Marseille Université), ses travaux de recherche se concentrent sur des problématiques orientées canal (logistique, SCM, distribution) portant sur le développement durable / la RSE, la qualité de service en $B 2 B$ et B2C et l'innovation. Ses expertises sectorielles incluent le packaging, l'informatique, les prestations logistiques, la grande distribution et l'enseignement supérieur (professeur depuis 2001 dans les champs disciplinaires logistique-SCM et marketing ; chaire du pôle marketing durant 7 ans). À son actif, il comptabilise une trentaine de publications nationales et internationales dans des revues scientifiques à comité de lecture, des actes de conférences, des ouvrages collectifs et des revues professionnelles.

\section{Références}

Bertolini, G. 1995. La double vie de l'emballage. Paris : Économica.

Bienstock, C. C., M. B. Royne, D. Sherrell, and T. F. Stafford. 2008. "An Expanded Model of Logistics Service Quality: Incorporating Logistics Information Technology." International Journal of Production Economics 113 (1) : 5-22.

Bonet-Fernandez, D., I. Petit, et A. Lancini. 2014. « L'économie circulaire : quelles mesures de la performance économique, environnementale et sociale ? " Revue Française de Gestion Industrielle 33 (4) : 23-43.

Boukouna, K. 2016. Citée par M. Rabiller. Dans « All4pack s'emballe pour la manutention. "Supply Chain Magazine 108 (octobre) : 48-49.

Brundtland, G. 1987. Our Common Future, Report of the World Commission on Environment and Development, Oslo, United Nations. New York: UN.

CNE. 2014. Emballages \& économie circulaire. Paris : Conseil National de l'Emballage.

CNE. 2015. L'emballage, acteur important de la logistique des produits. Paris : Conseil National de l'Emballage.

Devismes, P. 2005. Packaging mode d'emploi - De la conception à la distribution. Paris : Dunod.

Duong, H. T., et G. Paché. 2015. "Théorie des ressources appliquée à la logistique : une identification de cinq dimensions clés. " Logistique \& Management 23 (2) : 55-71.

Ellen MacArthur Foundation. 2013. Towards the Circular Economy: Opportunities for the Consumer Goods Sector, $112 \mathrm{pp}$. https://www.ellenmacarthurfoundation.org (consulté le 7 mai 2016).

Fontaine, M. 2016. L'Emballage, ce bel inconnu. Paris : Édition BoD - Book on Demand.

Freeman, E. 1984. Strategic Management: A Stakeholder Approach. Boston, MA: Pitman.

Fulconis, F., G. Paché, et E. Reynaud. 2016. "Vers une nouvelle forme de croissance économique - Les apports des recherches en logistique et supply chain management. » Revue Française de Gestion 42 (261, NovembreDécembre) [à paraître].

Gammelgaard, B., and P. D. Larson. 2001. "Logistics Skills and Competencies for Supply Chain Management." Journal of Business Logistics 22 (2): 27-50.

García-Arca, J., and J. C. Prado Prado. 2008. "Packaging Design Model From A Supply Chain Approach." Supply Chain Management: An International Journal 13 (5): 375-380.
Gold, S., S. Seuring, and P. Beske. 2010. "Sustainable Supply Chain Management and Inter-Organizational Resources: A Literature Review." Corporate Social Responsibility and Environmental Management 17 (4): 230-245.

Govindan K., H. Soleimani, and D. Kannan. 2015. "Reverse Logistics and Closed-loop Supply Chain: A Comprehensive Review to Explore the Future." European Journal of Operational Research 240 (3): 603-626.

Hamel, G., and C. K. Prahalad. 1990. "The Core Competence of the Corporation." Harvard Business Review 68 (3): 79-93.

Hellström, D., and M. Saghir. 2007. "Packaging and Logistics Interactions in Retail Supply Chains." Packaging Technology and Science 20 (3): 197-216.

Jahre, M., and C. J. Hatteland. 2004. "Packages and Physical Distribution." International Journal of Physical Distribution \& Logistis Management 34 (2): 123-139.

Johnsson, M. 1998. "Packaging Logistics: A Value-Added Approach." Doctoral thesis, Department of Engineering Logistics, Lund University, Lund.

Kaplan, R. S., and D. P. Norton. 1992. "The Balanced Scorecard: Measures that Drive Performance." Harvard Business Review 70 (1): 71-79.

Kaplan R. S., and D. P. Norton. 1996. The Balanced Scorecard: Translating Strategy into Action. Boston, MA: Harvard Business School Press.

Le Moigne, R. 2014. L'Économie circulaire - Comment la mettre en œuvre dans l'entreprise grâce à la reverse supply chain ? Paris : Dunod.

Massaroni, E., A. Cozzolino, and E. Wankowicz. 2014. "Sustainable Supply Chain Management Needs Sustainable Packaging An Exploratory Study." Atti del XXVI Convegno annuale di Sinergie, Cassino, Université de Cassino.

McDonough, W., et M. Braungart. 2012. Cradle to cradle : créer et recycler à l'infini. Paris : Éditions Alternatives.

Mentzer, J.T., Flint, D. J. and G. T. Hult. 2001. "Logistics Service Quality as a Segment-Customized Process." Journal of Marketing 65 (October): 82-104.

Molina-Besch, K. and H. Pålsson. 2016. "A Supply Chain Perspective on Green Packaging Development - Theory vs Practice." Packaging Technology \& Science 29 (1): 45-63.

Nilsson F., A. Olsson, and F. Wikström. 2011. "Toward Sustainable Goods Flows: A Framework from a Packaging Perspective." In Proceedings of the $23^{\text {rd }}$ Nofoma Conference, Harstad, Norway, 17 pp. Lund: Lund University Publications.

Olsmats, C., and C. Dominic. 2003. "Packaging Scorecard: A Packaging Performance Evaluation Method." Packaging Technology and Science 16 (1): 9-14.

Park J., Sarkis, J. and Z. Wu. 2010. "Creating Integrated Business and Environmental Value within the Context of China's Circular Economy and Ecological Modernization." Journal of Cleaner Production 18 (15): 1494-1501.

Pratsch, L., and I. Ustad. 1996. Guide to Balanced Scorecard Performance Management Methodology. Washington, DC: Procurement Executives' Association.

Prévot, F., F. Brulhart, et G. Guieu. 2010. "Perspectives fondées sur les ressources. Proposition de synthèse. " Revue Française de Gestion 36 (204) : 87-103.

Rundh, B. 2005. "The Multi-faceted Dimension of Packaging." British Food Journal 107 (9): 670-684.

Sarkis, J. 2003. "A Strategic Decision Framework for Green Supply Chain Management." Journal of Cleaner Production 11 (4): 397-409. 
Schenkel, M., M. Caniëls, H. Krikke, and E. van der Laan. 2015. "Understanding Value Creation in Closed Loop Supply Chains: Past Findings and Future Directions." Journal of Manufacturing Systems 37 (3): 729-745.

Simms, C., and P. Trott. 2010. "Packaging Development: A Conceptual Framework for Identifying New Product Opportunities." Marketing Theory 10 (4): 397-415.

Souza, G. 2013. "Closed-Loop Supply Chains: A Critical Review, and Future Research." Decision Sciences 44 (1): 7-38.

Vachon, S., and R. D. Klassen. 2006. “Extending Green Practices across the Supply Chain: The Impact of Upstream and Downstream Integration." International Journal of Operations \& Production Management 26 (7): 795-821.

Verghese, K. and H. Lewis. 2007. "Environmental Innovation in Industrial Packaging: A Supply Chain Approach." International Journal of Production Research 45 (18-19): 4381-4401.
Wever, R. 2011. "Design for Volume Optimization of Packaging for Durable Goods." Packaging Technology and Science 24 (4): 211-222.

World Economic Forum. 2015. Beyond Supply Chains: Empowering Responsible Value Chains. Cologny/Genève: World Economic Forum.

Woronoff, D. 2014. Histoire de l'emballage en France du XVIII siècle à nos jours. Valenciennes : Presses Universitaires de Valenciennes (PUV).

Zhu, Q., Y. Geng, and K. H. Lai. 2010. "Circular Economy Practices among Chinese Manufacturers Varying in Environmental-Oriented Supply Chain Cooperation and the Performance Implications." Journal of Environmental Management 91 (6): 1324-1331.

Zhu, Q., J. Sarkis, and K.-H. Lai. 2008. "Green Supply Chain Management Implications for 'Closing the Loop'." Transportation Research Part E: Logistics and Transportation Review 44 (1): 1-18. 